\title{
Chromosome Pairing at Pachytene and Meiosis in Autotetraploid Rice
}

\author{
V. R. Reddi and T. V. V. Seetharami Reddi \\ Department of Botany, Andhra University, Waltair, India
}

Received June 28, 1975

Tetraploids of cultivated rice have been studied by many authors with the ultimate aim of practical utilization. Although a general reduction in vigour is noticed, increase in size of some individual parts is a characteristic feature of the rice tetraploids (Ramiah et al. 1935, Morinaga and Fukushima 1937, Beachell and Jones 1945, Cua 1950, Oka 1953, Ghosh 1967). Induced tetraploids are also reported in wild species of Oryza (Gopalakrishnan and Shastry 1964). In general, tetraploids of $F_{1}$ hybrids between interracial and interspecific crosses and the progenies from irradiated tetraploids have shown considerable increase in spikelet fertility than autotetraploids (Richharia and Govindaswami 1963). Segregates upto $75 \%$ spikelet fertility were reported by these authors. Amphidiploids involving $O$. sativa, $O$. glaberrima and $O$. breviligulata have shown $75 \%$ pollen fertility and $12.72-47 \%$ spikelet fertility while the corresponding undoubled $F_{1}$ hybrids are observed to be completely sterile (Gopalakrishnan et al. 1964). Induced tetraploids reported in literature are usually of sectorial type. In spite of these studies, the possibilities for the exploitation of autotetraploids do not appear to have been fully explored in rice (cf. Nayar 1973). Obviously, there is still much scope in the study of induced polyploids of both cultivated varieties and also those produced from intervarietal and interspecific hybrids.

The present report deals with successful attempts on colchicine induced autotetraploids of Sona, one of the popular high yielding varieties of Andhra Pradesh, India, with long slender grains. The study is especially prompted by the possibility of obtaining relatively well spread pachytene cells in diploid and autotetraploid lines. Although the feasibility of studying the detail morphology of rice chromosomes at pachytene has been demonstrated by Shastry and his colleagues (cf. Shastry 1964) following Yao et al. (1958), such studies are not extended systematically to tetraploids. Pairing in autotetraploids at pachytene is competitive and is limited to sets of four homologous chromosomes. At any one location only two of the four homologues can conjugate but there could be exchanges of partners in a given association which may lead to multivalent formation at diakinesis and metaphase I provided the requisite number of chiasmata are formed at appropriate places.

Materials and methods

Three day old seedlings of Sona (IET1991) are treated with $0.1 \%$ aqueous 
solution of colchicine for 5, 7 and $9 \mathrm{hrs}$ under laboratory conditions. The seedlings are kept in an inverted position in petri dishes with the aid of a wire mesh. The tips of the plumules of the seedlings are excised to facilitate the penetration of colchicine solution. Care is taken to prevent desiccation of roots by covering them with moist blotting paper. After treatment, the seedlings are washed in running water for 30 minutes and were allowed to recover for $12 \mathrm{hrs}$ and then grown in specially prepared pots kept in green house. In order to stimulate growth and hence to increase the survival rate the seedlings are sprayed with $25 \mathrm{ppm}$ gibberellic acid on alternative days for three weeks.

A single plant with entirely polyploid shoot was obtained only in the lot treated for $9 \mathrm{hrs}$ period. For cytological study immature spikelets are fixed in $1: 4$ acetic alcohol to which a trace of ferric chloride is added. Fixation is effected at $14^{\circ} \mathrm{C}$ for $24 \mathrm{hrs}$ after which the material was stored in $70 \%$ alcohol until used for study. Smear preparations of anthers were made in $1 \%$ acetocarmine.

Seed has been collected from a total of 19 productive tillers of the tetraploid plant. In the $\mathrm{C}_{2}$ population a large number of plants reverted to diploid condition. Only three tetraploid plants could be located in a total population of 80 plants.

\section{Results}

Induction of a complete tetraploid through colchicine is considered to be of very low probability in rice. The occurrence of such a plant reported herein may be attributed to gibberellic acid spraying on treated seedlings. The tetraploid plant resembled the diploid and is even less vigourous but marked increase in size of glume, lodicule and anthers of the spikelet is found. The tendency for development of awn is also noticeable. Increase in pollen grain size, stomata and of the grain is evident (Table 1). The pollen fertility in diploid is $79.0 \%$ but in the tetra-

Table 1. Comparison of stomata and fertility characteristics of diploid and autotetraploid

\begin{tabular}{|c|c|c|c|c|c|c|}
\hline \multirow{2}{*}{ Plant type } & \multicolumn{2}{|c|}{ Stomata } & \multicolumn{2}{|c|}{ Pollen } & \multicolumn{2}{|c|}{ Spikelet } \\
\hline & $\begin{array}{l}\text { Length } \\
(\mu)\end{array}$ & $\begin{array}{l}\text { Breadth } \\
\quad(\mu)\end{array}$ & $\begin{array}{l}\text { Diameter } \\
(\mu)\end{array}$ & $\begin{array}{c}\text { Fertility } \\
(\%)\end{array}$ & $\begin{array}{l}\text { Length } \\
\text { (mm) }\end{array}$ & $\begin{array}{c}\text { Breadth } \\
\text { (mm) }\end{array}$ \\
\hline \multirow[t]{2}{*}{$2 \mathrm{n}$} & 23.47 & 15.02 & 34.10 & 79.0 & 8.80 & 1.84 \\
\hline & \pm 2.06 & \pm 1.47 & \pm 3.23 & & \pm 1.07 & \pm 0.18 \\
\hline \multirow[t]{2}{*}{$4 n$} & 24.76 & 17.38 & 43.98 & 53.3 & 9.85 & 2.32 \\
\hline & \pm 2.03 & \pm 1.88 & \pm 4.55 & & \pm 0.57 & \pm 0.27 \\
\hline
\end{tabular}

\pm indicates standard deviation value.

ploid plant it was only $53.3 \%$. The number of spikelets produced on the tetraploid panicle is reduced when compared to the diploid.

Considerable effort in making the smear preparation and careful screening of PMCs yielded pachytene cells in the diploid of the type depicted in Fig. 1. Although the end to end tracing of the 12 pachytene bivalents could be achieved in such cells, the location of centromere positions is not always certain in some cases 

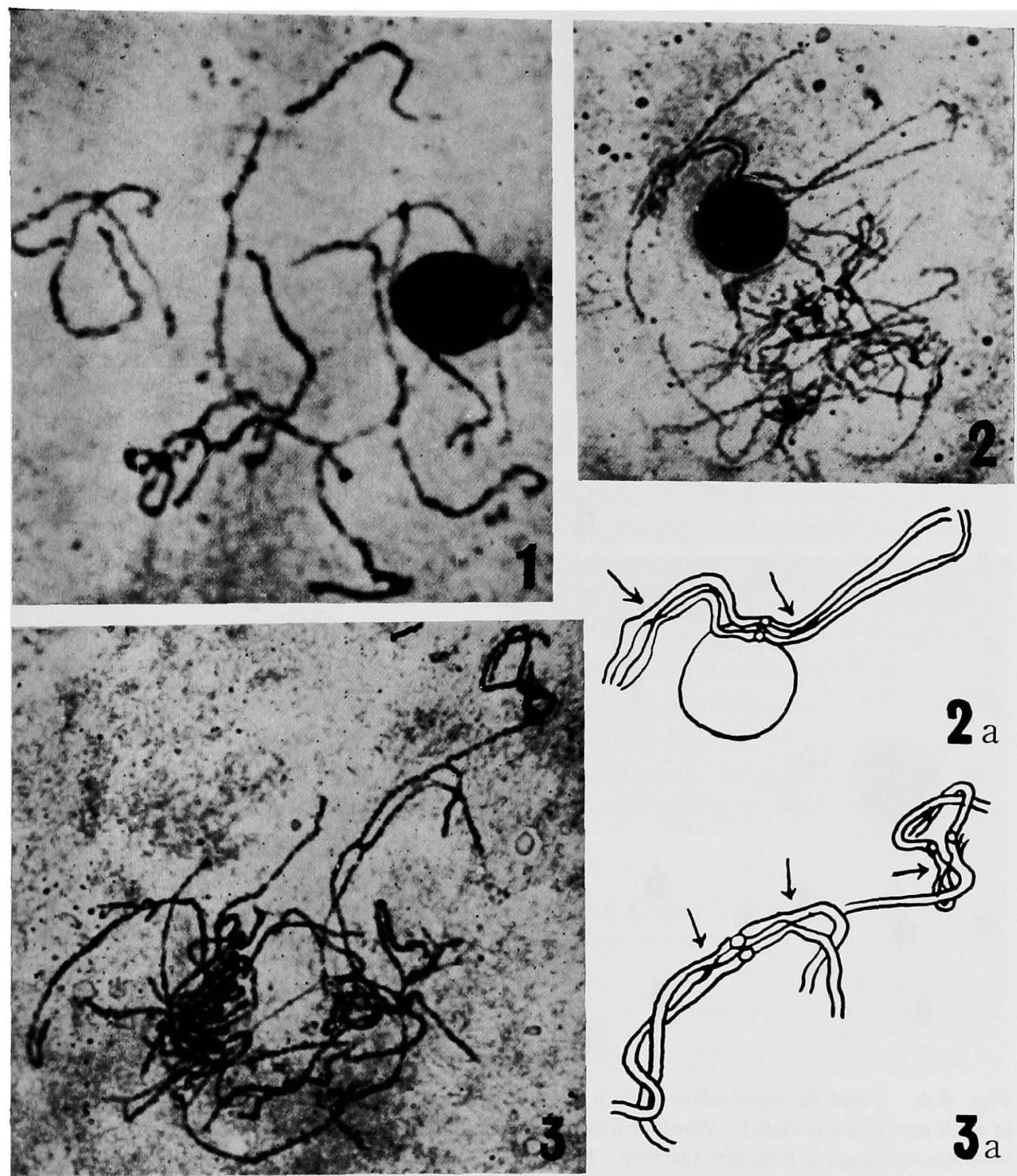

Figs. 1-3. 1, pachytene in diploid. $\times 1500$. 2-6. meiosis in autotetraploid. $\times 1000.2$ and 2 a, pachytene showing an association of four homologues of the longer nucleolar chromosome with two exchanges one in each arm (arrows). 3 and 3a, pachytene showing an association of four in one of the shorter chromosomes with one exchange in the long arm (upper right). Association of the longest chromosome (No. 1) can be seen with two exchanges inidcated arrows (middle right).

which makes the comparison of particular chromosomes in different cells somewhat arbitrary. Absence of cytologically recognizable landmarks on the chromosomes of rice adds to the difficulty of identification. In general, the pachytene chromosomes of Sona have an average length varying from 43.9 microns of the longest to 20.7 microns of the shortest in the complement. The second and third chromosomes with 36.3 and 34.6 microns average length together with the longest can be considered to constitute a group of longer chromosomes with submedian centro- 


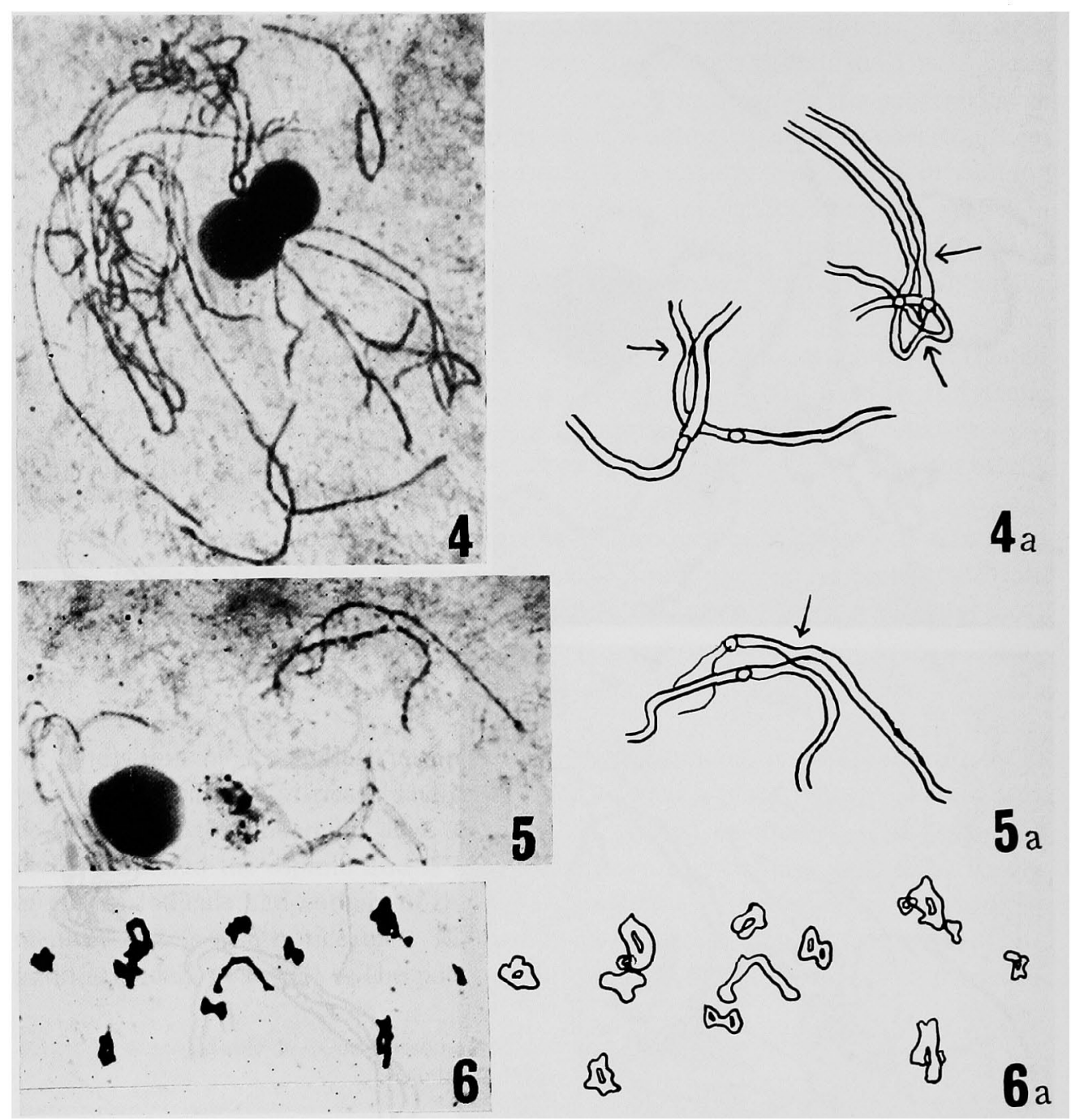

Figs. 4-6. 4 and 4a, association of four homologues of chromosome 2 with two exchanges one in each arm (middle right). Another association of four homologues of probably chromosome 5 with one exchange in long arm (arrow). Note the deep stained chromomeres terminating the short arms. 5 and $5 \mathrm{a}$, association of four homologues of chromosome 3 with one exchange in long arm (arrow). Note the short arm terminal unpaired region. 6 and 6a, metaphase I with 10 IV (types $11,13,17$ and 18 and $4 \mathrm{II})$.

meres. Two others are found attached to the nucleolus with the shorter one having an average length of 20.7 microns and showed terminal attachment. The centromere is subterminal and this leads to frequent rod type bivalent formation. The longer nucleolar chromosome measured 30.6 microns and had subterminal attachment. The centromere in this case appears to be submedian and the bivalent realized at diakinesis is of ring type. The remaining 7 chromosomes of the complement showed a gradual decrease in total length (average means are 36.6, 31.9, $31.6,29.6,26.9,25.3$ and 23.6 microns) and probably carry submedian centromeres with the possible exception of one with a subterminal centromere. There is a close 
resemblance in the morphology of the pachytene chromosomes of Sona to those of IR8 variety in which induced single and multiple interchanges have been isolated (Reddi and Reddi 1975).

At early diakinesis a maximum of 3 bivalents showed 3 chiasmata each which are presumably formed by the 3 longer chromosomes. The rest of the chromosomes commonly formed ring type bivalents. Usually a single rod bivalent and less frequently two are found at diakinesis and metaphase I. The average frequency of chiasmata at metaphase $I$ is observed to be 22.2 based on the analysis of 30 cells. Only 12.7 chiasmata are terminalized and the rest of them remained interstitial.

The occurrence of four homologues for each of the 12 chromosomes in autotetraploid complicates the pairing process. Stages earlier to pachytene are not amenable for analysis. At pachytene it was observed that pairing at any location can take place between two of the four chromosomes and this leads to partner exchanges. Only one or more associations of four can be studied in single cells. A total of 30 associations representing many of the chromosomes in the complement could only be studied. There could be two separate bivalents formed for a given chromosome or alternatively the association of four when realized showed one or two exchanges. Relative to the arm lengths there appears to be even distribution of exchanges (Figs. 2, 3 and 4). The initial points of contract in the process of pairing appear to be located in the regions adjoining centromeres. This is borne out by the occasional failure of pairing in terminal regions of chromosomes as shown in Fig. 5. It has been recognized that partner exchanges are a prerequisite for multivalent associations at diakinesis provided chiasma formation takes place at appropriate locations.

Table 2. Chromosome associations in autotetraploid at metaphase I

\begin{tabular}{|c|c|c|c|c|}
\hline \multicolumn{3}{|c|}{ Association } & \multirow{3}{*}{ Frequency } & \multirow{3}{*}{$\begin{array}{c}\text { Total } \\
\text { chiasmata } \\
\text { per cell }\end{array}$} \\
\hline \multirow{2}{*}{$\begin{array}{l}\text { Number of } \\
\text { quadrivalents }\end{array}$} & \multicolumn{2}{|c|}{ Bivalents } & & \\
\hline & Rings & Rods & & \\
\hline 6 & 9 & 3 & 2 & 45 \\
\hline 7 & 7 & 3 & 1 & 43 \\
\hline 7 & 6 & 4 & 1 & 42 \\
\hline 7 & 5 & 5 & 1 & 42 \\
\hline 7 & 4 & 6 & 1 & 41 \\
\hline 8 & 8 & - & 2 & 47 \\
\hline 8 & 7 & 1 & 2 & 47 \\
\hline 8 & 5 & 3 & 4 & 43 \\
\hline 8 & 4 & 4 & 2 & 41 \\
\hline 8 & 3 & 5 & 2 & 41 \\
\hline 9 & 6 & - & 1 & 49 \\
\hline 9 & 5 & 1 & 1 & 47 \\
\hline 10 & 4 & - & 2 & 46 \\
\hline
\end{tabular}

At metaphase I, a total of 22 cells have been analyzed and the data are summarized in Table 2. Only quadrivalents and bivalents are observed and univalents and trivalents are completely absent. The frequency of quadrivalents varied from 
a minimum of 6 to a maximum of 10 with an average of 7.9 per cell (Fig. 6). The ring and rod type bivalents averaged 5.4 and 2.5 respectively. Although quadrivalent types $11,12,13,14,15,16,17$ and 18 are found, only the chain and ring types predominated (Darlington 1965). The orientation of quadrivalents at metaphase I could be alternate, adjacent, linear or indifferent. All the same, the anaphase I segregations are numerically balanced in majority of the cells examined. One or two lagging chromosomes and unequal distributions were observed to a limited extent. However, the observed reduction in pollen fertility and seed set cannot be accounted on the basis of the limited number of unbalanced gametes and zygotes that may be formed. Obviously genetic factors are of importance in tetraploid rice in this regard.

\section{Discussion}

Post-pachytene observations made in the present study of autotetraploid rice are similar to the previous reports. Ichijima (1934) reported 5-6 quadrivalents and 10-12 bivalents at meiosis. The average quadrivalent frequency of 8.93 per cell reported by Cua (1950) is slightly higher than the present report of 7.90. Ghosh (1967) however, obtained an average value of 7.46 per cell which is nearer to the value in the present material. Bouharmont (1963) reported 63-78\% of the chromosomes in the tetraploid rice associated in quadrivalents at diakinesis. According to him, the low chiasma frequency, the increase in number of univalents and trivalents at metaphase I, followed by decrease in quadrivalent fromation, suggest that some of the quadrivalents are false and nonchiasmatic. In the present study, no valid evidence could be obtained for the occurrence of false quadrivalents. On the other hand, $65.83 \%$ of the chromosomes at metaphase I associated into quadrivalents in the present material which is in conformity with the findings of Morrison and Rajhathy (1960) who observed that tetraploid plants with shorter chromosomes formed even more quadrivalents than those with longer chromosomes and that approximately $2 / 3 \mathrm{~s}$ of the chromosomes associate in the quadrivalents in all cases.

A slight reduction in quadrivalent frequency after 8 generations of selection for improved fertility in autotetraploids was observed by Richharia and Govindaswami (1963). Increased fertility in intervarietal tetraploids was generally found when compared to diploid $F_{1}$ hybrids (Morinaga and Kuriyama 1946, Hsieh 1952, Oka 1954, Majima et al. 1958). Cua (1952) observed lower quadrivalent frequency in indica-japonica intervarietal tetraploid hybrids and felt that chromosome differentiation and heterozygosity was the cause of increase in fertility and that this would make possible to select superior tetraploid types. Oka (1955) however, attributed the increase in fertility to complementary action of genes.

Reduction in pollen fertility and seed set in tetraploid is attributed to irregular distribution of chromosomes in multivalents (Darlington 1965). Hazarika and Rees (1967) working with rye autotetraploids observed that in general, with a higher chiasma frequency the number of quadrivalents increases. The data presented in Table 2 reveals that such a relation does not hold good in the tetraploid 
rice of the present study. Apart from the frequency and types of quadrivalents the relative frequency of ring and rod type bivalents also determine the chiasma frequencies of different cells analyzed. The average value of 44.0 per cell obtained in the tetraploid of the present study is almost double the value of 22.2 recorded in the comparable diploid.

It is more likely, that tetraploids of hybrid origin breed true to the chromosome number and if a wide range of intra and interspecific tetraploids are produced it might be possible to develop completely fertile lines. The study of pairing relationships at pachytene in segregates with improvement in fertility will add precision to the understanding of cytological situations which increase the fertility levels. Likewise, a better appraisal of genome relationships and chromosome differentiation could be made in hybrid tetraploids through the study of pairing relationships of pachytene chromosomes.

\section{Acknowledgements}

We are thankful to Prof. J. V. Pantulu, Head of the Department of Botany, Andhra University, Waltair, for facilities. The junior author is grateful to the Council of Scientific and Industrial Research for financial assistance.

\section{Literature cited}

Beachell, H. M. and Jones, J. M. 1945. Tetraploids induced in rice by temperature and colchicine treatments. J. Amer. Soc. Agron. 37: 165-175.

Bouharmont, J. 1963. Pseudo-quadrivalents in an autotetraploid rice. Nature (London) 197: $410-411$.

Cua, L. D. 1950. Artificial polyploidy in the oryzeae II. On the germination behaviour of diploid and autotetraploid rice (Oryza sativa L.). Nippon Idengaku Zasshi 25: 161165.

- 1952. Artificial polyploidy in the oryzeae III. Cytogenetical studies on intra- and intersubspecies tetraploid hybrids in Oryza sativa L. Seiken Ziho 5: 42-53.

Darlington, C. D. 1965. Recent Advances in Cytology. 3rd ed. Churchill, London.

Ghosh, M. 1967. Induction of polyploidy in rice through colchicine. Nucleus (Calcutta) 10: 194-207.

Gopalakrishnan, R. and Shastry, S. V.S. 1964. Induced polyploidy in Oryza officinalis Wall ex Watt and $O$. australiensis Domin. Curr. Sci. 33: 504.

-, Nayar, N. M. and Sampath, S. 1964. Cytogenetical studies of two amphidiploids in the genus Oryza. Euphytica 13: 57-64.

Hazarika, M. H. and Rees, H. 1967. Genotypic control of chromosome behaviour in rye X. Chromosome pairing and fertility in autotetraploids. Heredity 22: 317-332.

Hsieh, S. C. 1952. Studies on the behaviour of the chromosomes in tetraploid rice. J. Agr. Res., Taipei 3(2): 1-15 (in Chin.).

Ichijima, K. 1934. On the artificially induced mutations and polyploid plants of rice occurring in subsequent generations. Proc. Imp. Acad. (Tokyo) 10: 388-391.

Masima, I., Sato, H. and Uchiyamada, H. 1958. Correlations between varietal hybrid sterility and tetraploid hybrid sterility in rice. Jap. J. Breed. 7: 141-144 (in Jap.).

Morinaga, T. and Fukushima, E. 1937. Cytogenetical studies in Oryza sativa L. III. Spontaneous autotetraploid mutants in Oryza sativa L. Jap. Bot. 9: 71-94.

- and Kuriyama, H. 1946. The effect of hybridization on the year by year improvement in the 
fertility of autotetraploid rice. Nippon Idengaku Zasshi 21: 81-83 (in Jap.).

Morrison, J. W. and Rajhathy, T. 1960. Chromosome behaviour in autotetraploid cereals and grasses. Chromosoma 11 : 297-309.

Nayar, N. M. 1973. Origin and cytogenetics of rice. Advan. Genet. 17: 153-292.

Oka, H. I. 1953. Studies on tetraploid rice I. Method of colchicine treatment in rice. Nippon Idengaku Zasshi 28: 227-232 (in Jap.).

- 1954. Studies on tetraploid rice IV. $F_{1}$ hybrids between tetraploid varieties of rice. Nippon Idengaku Zasshi 29: 101-108 (in Jap.).

- 1955. Studies on tetraploid rice VI. Fertility variation and segregation ratios for several characters in tetraploid rice $O$. sativa $L$. Cytologia 20: 258-266.

Ramiah, K., Parthasarathy, N. and Ramanujam, S. 1935. A tetraploid plant in wild rice Oryza longistaminata. Proc. Indian Acad. Sci., Sect. B1 : 565-570.

Richharia, A. K. and Govindaswamy, S. 1963. Selection for spikelet fertility in induced tetraploid rices. Sci. and Cult. 29: 560-562.

Reddi, V. R. and Reddi, T. V. V.S. R. 1975. A case of induced double interchange in rice involving six chromosomes. Mutation Res. (submitted for publication).

Shastry, S. V. S. 1964 . New approach to study of rice karyomorphology. In "Rice Genetics and Cytogenetics". Proc. Symp., Los Banos, Philippines, 1963, pp. 62-67. Elsevier, Amsterdam.

Yao, S. Y., Henderson, M. T. and Jodon, N. E. 1958. Cryptic structural hybridity as a probable cause of sterility in intervarietal hybrids of cultivated rice $O$. sativa $\mathrm{L}$. Cytologia 23: 46-55. 